Review Article

\title{
Effect of Tongqiao Huoxue Decoction Combined with Western Medicine on Ischemic Stroke: A Systematic Review
}

\author{
Da-yuan Zhong $\mathbb{D}^{1},{ }^{1}$ Hao-yue Li, ${ }^{1,2}$ Lan Li, ${ }^{1}$ Ruo-meng Ma, ${ }^{1}$ Cheng-ting Jiang, \\ Ding-xiang Li $\mathbb{1},^{1}$ and Yi-hui Deng $\mathbb{1}^{1}$ \\ ${ }^{1}$ Hunan University of Chinese Medicine, Changsha 410208, China \\ ${ }^{2}$ Hunan Provincial Brain Hospital, Changsha 410208, China \\ Correspondence should be addressed to Ding-xiang Li; ldxlzy@hotmail.com and Yi-hui Deng; 644138330@qq.com
}

Received 8 August 2020; Revised 8 November 2020; Accepted 19 November 2020; Published 16 December 2020

Academic Editor: Wen yi Kang

Copyright (c) 2020 Da-yuan Zhong et al. This is an open access article distributed under the Creative Commons Attribution License, which permits unrestricted use, distribution, and reproduction in any medium, provided the original work is properly cited.

\begin{abstract}
Objective. We conducted a systematic review and meta-analysis to systematically evaluate the curative effect of Tongqiao Huoxue Decoction (TQHXD) combined with Western medicine treatment (WMT) on Ischemic Stroke (IS). Methods. Randomized controlled trials (RCTs) of TQHXD in the treatment of IS by computer retrieval of PubMed, Embase, Web of Science, Chinese Biomedical Literature Service System, CNKI, Wanfang Database, and Weipu Database. The retrieval time was taken from the establishment of the database to July 30, 2020. Two researchers, respectively, conducted a strict evaluation of the quality of the literature and extracted the data which were then entered in the RevMan5.3 software for meta-analysis. Results. 40 articles were listed, which involved 3260 patients. Meta-analysis results show that TQHXD combined with WMT can significantly reduce patients' NIHSS score, serum hypersensitivity C-reactive protein (hs-CRP), plasma viscosity, serum fibrinogen, serum total cholesterol, and serum triglycerides and improve patients' ADL-Barthel scoring and treatment efficiency. However, there is no evidence that TQHXD combined with the WMT group can significantly decrease the incidence of adverse events. Conclusion. The therapeutic effect of TQHXD combined with the WMT group was significantly better than that of the WMT alone group. For the treatment of patients with IS, TQHXD combined with WMT is worthy of application and promotion.
\end{abstract}

\section{Introduction}

Depending on the latest report from the World Health Organization, stroke is the second leading cause of death worldwide [1]. In 2016 alone, approximately 5.5 million people died of a stroke [2]. China is one of the countries with the heaviest stroke burden in the world. The latest census results showed that the incidence of stroke disease in China is about $1.6 \%$, of which Ischemic Stroke (IS) accounts for $21.4 \%$ [3]. Evidence-based medicine research results [4] showed that most ischemic strokes are caused by blood clots blocking cerebral blood vessels. Elevated plasma fibrinogen is an important pathogenic factor of cerebrovascular diseases [5], and its increased content will cause plasma viscosity to rise, increase red blood cell and platelet aggregation, increase whole blood viscosity, and promote thrombosis, which leads to ischemia Sexual events. Bushi's studies have shown that thrombin activity is generally enhanced in patients with ischemic cerebrovascular disease and their high-risk groups [6]. Animal experiments [7] found that in the ischemic center of the rat model of middle cerebral artery occlusion, the activity of thrombin was considerably increased, and the expression of the prothrombin gene was upregulated. In vitro experiments [8] have shown that the nonproteolytic activity of thrombin could activate microglia and enhance microglia phagocytosis, thereby causing damage to neurons [9].

According to the symptoms and manifestations of IS, Chinese medicine believes that its onset is mainly caused by poor blood circulation $[10,11]$, so treatments that promote blood circulation are typically used for treatment. Tongqiao Huoxue Decoction (TQHXD) is derived from Wang 
Qingren's book Medical Forest Correction. Wang believed that it has the effects of promoting blood circulation, relieving pain, and refreshing. Studies [12] found that highdose TQHXD could significantly improve the neuromotor function of rats with IS, increase serum NO and ET-1 levels, and reduce brain tissue Glu and Asp levels. TQHXD [13] may alleviate the neurological deficit in rats with cerebral ischemia and reperfusion by promoting the expression of neurotrophic factors in brain tissue and inhibiting lipid peroxidation. It can be seen that by looking for a suitable entry point, TQHXD combined with Western medicine treatment (WMT) can exert a beneficial effect. At present, a large number of clinical trials have confirmed the clinical efficacy of TQHXD in the treatment of IS. However, due to the different quality and sample size of the research, it lacks strong convincing power and lacks evaluation research on systematic safety and effectiveness. In order to provide an evidence-based reference for clinical medication, this article collects the published literature of TQHXD for the treatment of IS to conduct a systematic review.

\section{Information and Methods}

2.1. Research Registrations. We log in to PROSPERO official website (https://www.crd.york.ac.uk/PROSPERO/) to register a protocol for meta-analysis; the number of successful registration is CRD42020147688.
2.2. Types of Studies Included in the Study. Only clinical randomized controlled trials (RCTs) of TQHXD combined with WMT in the treatment of IS are included.

2.3. Research Objects Included in the Study. Patients with general clinical signs and symptoms of IS, excluding other diseases [14], are included. The course of the disease is within 2 weeks.

2.4. The Main Intervention Measures Are Included in the Study. The treatment group was TQHXD combined with the WMT group, the control group was the WMT group, and the two groups were both treated without acupuncture, cupping, massage, and other rehabilitation methods.

2.5. Outcome Indicators Are Included in the Study. We mainly extract the patient's NIHSS score, ADL-Barthel score, hsCRP (high-sensitivity C-reactive protein), plasma viscosity, fibrinogen, TC (total cholesterol), TG (triglycerides) before and after treatment, the treatment effective rate, and the incidence of adverse reactions. The calculation method [15] of the change of the mean value and the standard deviation is as follows, where $R$ is a constant, and $R$ is 0.5 in this study.

$$
\begin{aligned}
\text { mean }(\text { change }) & =\text { mean }(\text { before })-\text { mean }(\text { after }) \\
\operatorname{sd}(\text { change }) & =\sqrt{\operatorname{sd}(\text { before })^{2}+\operatorname{sd}(\text { after })^{2}-2^{*} R^{*} \text { sd }(\text { before })^{*} \text { sd }(\text { after })} .
\end{aligned}
$$

2.6. Research Exclusion Criteria. The exclusion criteria are as follows: the type of study does not correspond to nonrandomized controlled study, the intervention measures of the study do not match, the study is repeated, and the study does not contain 9 kinds of outcome indicators.

2.7. Retrieval Methods and Strategies. We search the following databases: PubMed database (https://pubmed.ncbi. nlm.nih.gov/), Embase database (https://www.embase.com), Web of Science database (http://webofscience.com), China Biomedical Document Service System (http://www. sinomed.ac.cn/), CNKI database (https://www.cnki.net/), Wanfang Database (http://www.wanfangdata.com.cn/index. html), and Weipu Database (http://www.cqvip.com/). Search terms: Stroke, Ischemic Stroke, Cerebral infarction, and Tongqiao Huoxue Decoction. Search time limit: database establishment to July 30, 2020. Search strategy: search terms are searched by free words and subject terms. Search strategy: search by free words and subject terms.

The search strategy in PubMed is given below.

\#1. Stroke [Title/Abstract]

\#2. Ischemic Stroke [Title/Abstract]

\#3. Cerebral Infarction [Title/Abstract]

\author{
\#4. Tongqiao Huoxue Decoction [Title/Abstract] \\ \#5. \#1 or \#2 or \#3 \\ \#6. \#4 and \#5
}

2.8. Data Extraction and Quality Evaluation. The documents retrieved from each database were entered into the CNKIEstudy software to eliminate duplicates, read the title and abstract according to the PICOS principle, and then read the full text and finally determined to be included in the study. The quality evaluation relates to the Cochrane risk bias assessment tool. The obtained documents are provided and the quality was evaluated by two staff members (Lan Li and Ruo-meng Ma). Any differences during the period will be arbitrated by a third member (Da-yuan Zhong).

2.9. Statistical Analysis. RevMan5.3 software was used for data analysis. Binary variables use Odds Ratio (OR) and Relative Risk (RR); continuous variables use standard deviation (MD) as effective indicators. Using $t^{2}$ and $\mathrm{Chi}^{2}$ statistics to analyze the heterogeneity between the studies, if $I^{2} \leq 50 \%$, it indicates that the heterogeneity between the studies is small, and then the fixed-effects model is used. 
Otherwise, it indicates that the heterogeneity is large, and the random-effects model is used.

\section{Results}

3.1. The Search Results and the Basic Characteristics of the Included Studies. A total of 40 RCTs meeting the criteria were included [16-55], involving 3260 patients. The literature search and inclusion flow chart is shown in Figure 1. The basic characteristics of the included studies are shown in Table 1.

3.2. Methodological Quality Evaluation. 14 studies $[16,17,20,21,26,29,30,33,35,38,40,43,46,48]$ mentioned specific random methods; all included studies [16-55]. No allocation concealment method or blinding method is mentioned, except for 2 studies $[29,36]$. The rest of the studies have complete data and no selective reports; it is not clear whether there are other biases, as shown in Figures 2(a) and 2(b).

\subsection{Meta-Analysis Results}

3.3.1. NIHSS Score. There are 18 studies $[16,17,20,25,27,29-31,34,37-41,44,45,50,51]$ that reported the NIHSS scoring index. The heterogeneity test results showed that there are significant differences in heterogeneity among the 18 studies, so the random-effects model was used for meta-analysis. The results (Figure 3) showed that the NIHSS score of the TQHXD combined with WMT group was significantly different from the group of WMT alone $(\mathrm{MD}=3.11,95 \% \mathrm{CI}(2.35,3.87), P<0.00001)$.

3.3.2. ADL-Barthel Score. There are 13 studies $[17,20,27,29,30,34,38-40,45,47,49,50]$ that reported the ADL-Barthel score index, and the heterogeneity test results showed that there are significant differences in heterogeneity among these 13 studies. Therefore, the random-effects model was used for meta-analysis, and the results (Figure 4) showed that the TQHXD combined with the WMT group was significantly different from the group of WMT alone $(\mathrm{MD}=11.07,95 \% \mathrm{CI}(5.89,16.25), P<0.00001)$.

3.3.3. Hypersensitive C-Reactive Protein. There are 7 studies $[18,21,28,30,31,43,47]$ that reported the index of highsensitivity C-reactive protein. The heterogeneity test showed that there are significant differences in heterogeneity among the 7 studies, so the random-effects model was used for meta-analysis. The results (Figure 5) showed that the TQHXD combined with the WMT group was significantly different from the group of WMT alone ( $\mathrm{MD}=1.89,95 \% \mathrm{CI}$ $(1.14,2.64), P<0.00001)$.

3.3.4. Plasma Viscosity. There are 9 studies $[21,23,26,30,33,34,38,43,46]$ that reported plasma viscosity indexes. The heterogeneity test results showed that there are significant differences in heterogeneity among these 9 studies, so the random-effects model was used for meta-analysis. The results (Figure 6) showed that the TQHXD combined with the WMT group was significantly different from the WMT group alone $(\mathrm{MD}=0.38,95 \% \mathrm{CI}$ $(0.21,0.55), P<0.00001)$.

3.3.5. Fibrinogen. There are 11 studies $[21,23,26,30,33,34,38,39,45-47]$ that reported fibrinogen indicators, and the heterogeneity test results showed that there are none significant differences in heterogeneity among these 11 studies, so the fixed-effects model was used for meta-analysis. The results (Figure 7) showed that the TQHXD combined with the WMT group was significantly different from the WMT group alone $(\mathrm{MD}=1.10,95 \% \mathrm{CI}$ (0.97, 1.23), $P<0.00001)$.

3.3.6. Serum Total Cholesterol. There are 4 studies $[19,25,26,28]$ that reported serum total cholesterol indicators. The heterogeneity test results showed that there are none significant differences in heterogeneity among the four studies. Therefore, a fixed-effects model was used for metaanalysis. The results (Figure 8) showed that the TQHXD combined with the WMT group was significantly different from the WMT group alone $(\mathrm{MD}=0.57,95 \% \mathrm{CI}(0.47,0.67)$, $P<0.00001)$.

3.3.7. Triglycerides. There are 5 studies $[19,25,26,28,30]$ that reported triglyceride indicators. The heterogeneity test results showed that there are significant differences in heterogeneity among the 5 studies. Therefore, a randomeffects model was used for meta-analysis. The results (Figure 9) showed that the TQHXD combined with the WMT group was significantly different from the WMT group alone $(\mathrm{MD}=0.47,95 \% \mathrm{CI}(0.22,0.71), P<0.00002)$.

3.3.8. Effectiveness of Treatment. There are 29 studies $[16,17,19,20,22-24,29-37,42-46,48-55]$ that reported effective rate indicators, and the heterogeneity test results showed that there are none significant differences in heterogeneity among these 29 studies, so a fixed-effects model was used for meta-analysis. The results (Figure 10) showed that the TQHXD combined with the WMT group was significantly different from the WMT group alone $(\mathrm{OR}=4.53,95 \% \mathrm{CI}(3.43,5.99), P<0.00001)$.

3.3.9. Incidence of Adverse Reactions. There are 10 studies $[17,20-22,25-27,29,30,42]$ that reported the incidence of adverse reactions. The heterogeneity test results showed that there are none significant differences in heterogeneity among these 10 studies, so the fixed-effects model was used for meta-analysis. The results (Figure 11) showed that the TQHXD combined with the WMT group and the WMT group were not significantly different from each other $(\mathrm{RR}=1.38,95 \% \mathrm{CI}(0.80,2.40), P=0.25)$. 

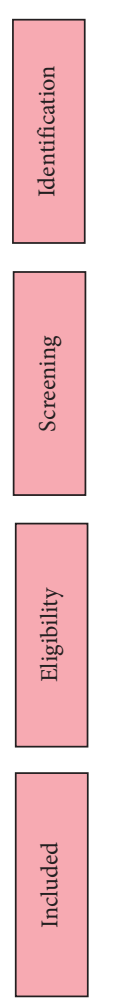

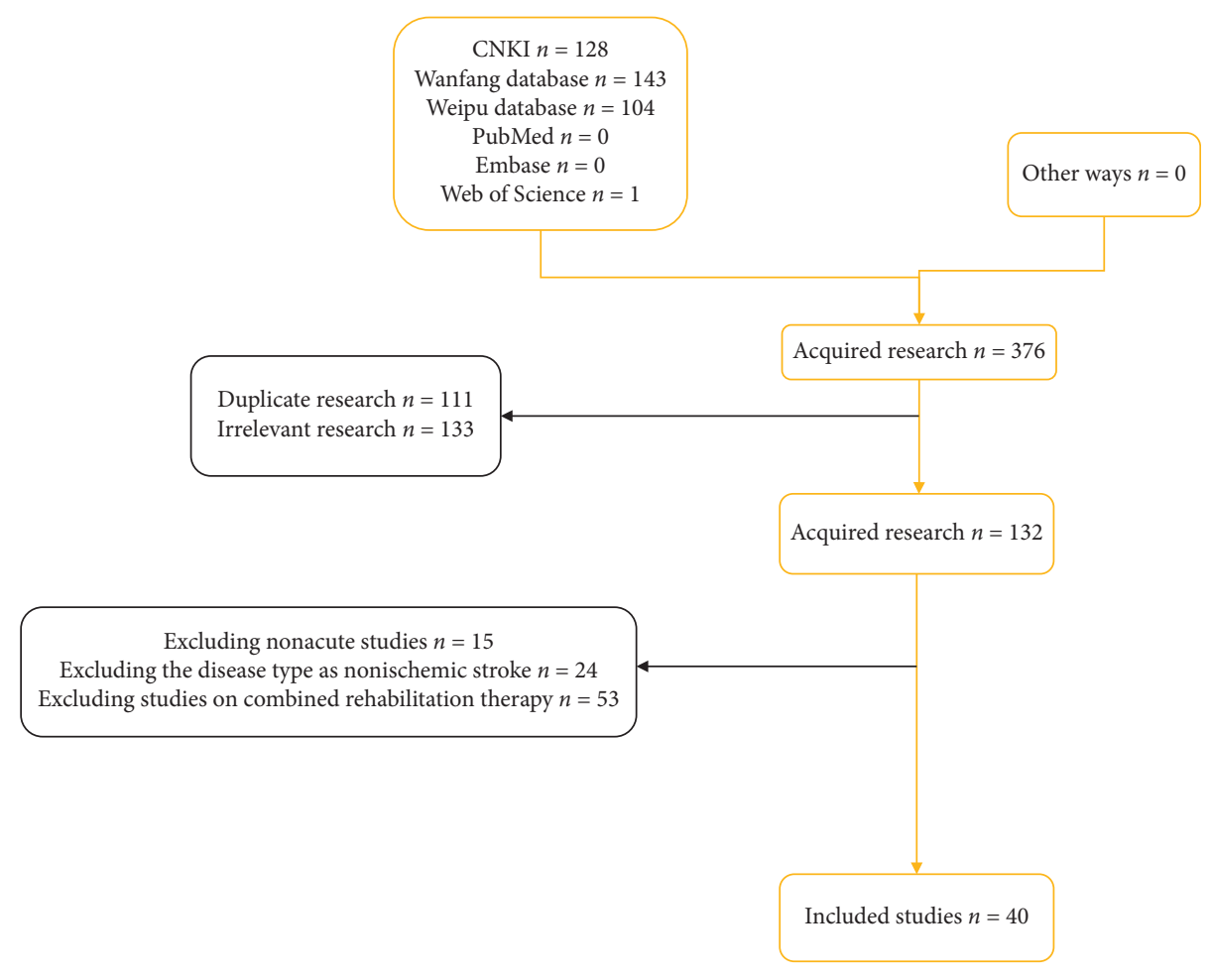

FIgURE 1: Flow chart of literature search inclusion.

3.4. Analysis of Publication Bias. The effectiveness of 29 studies $[16,17,19,20,22-24,29-37,42-46,48-55]$ was evaluated by the funnel chart, and the results showed that the funnel chart has good symmetry, as shown in Figure 12, which means that there is no publication bias in the study.

\section{Discussion}

IS is a common cause of death and acquired disability [56]. Its pathogenesis is mainly due to slow blood flow, changes in blood composition, and increased blood viscosity on the basis of arteriosclerosis. The key factor for treatment is improving blood circulation in the ischemic area as soon as possible, eliminating secondary edema, and restoring the normal metabolism and nerve function of brain cells. The current treatment for this IS is mainly through intravenous drug thrombolysis, arterial thrombolysis, nonstent mechanical thrombectomy, and stent mechanical thrombectomy treatment [57]. Among them, intravenous drug thrombolytic treatment time is stricter $(3-4.5 \mathrm{~h}$ in acute cerebrovascular occlusion), the recanalization rate of large artery occlusion is low, and the treatment effect is relatively poor. The cerebral infarction secondary to acute cerebrovascular occlusion is mostly caused by the formation of a large number of thrombi on the basis of cerebral artery stenosis. Even if the blood vessel is unblocked again after simple thrombolysis, the problem of obvious vascular stenosis will remain, and reocclusion is prone to occur [58]. Moreover, thrombolytic drugs could easily cause intracranial hemorrhage. The incidence of intracranial hemorrhage by intravenous thrombolysis is about $6.4 \%$ and that of arterial thrombolysis is close to $10 \%$. This makes the condition worse, so the effect of treatment is not particularly ideal $[59,60]$. Therefore, it is necessary to find more effective drugs to treat it. After thousands of years of development, Chinese medicine has left many classic prescriptions such as TQHXD and Buyang Huanwu Decoction, which have been used in the clinical treatment of human IS. Traditional Chinese medicine believes that the onset of IS is closely related to poor blood circulation and that blood stasis is both a pathological product and a pathogenic factor [11]. Modern physicians' understanding of the pathogenesis of IS by blood stasis and treatment methods for promoting blood circulation has become unified [61]. Poor blood circulation is considered to be the main cause of the disease, and the clinical application of the blood circulation method is effective. Significantly. TQHXD has the effects of promoting blood circulation, relieving pain, and resuscitating. It has been widely used in the treatment of brain injury diseases such as IS.

Meta-analysis is a statistical method that combines the effect size of the included studies of the same kind into a quantitative indicator. The combination can increase the sample size and improve the effectiveness of the test. Based on the meta-analysis method, this study combined 40 RCTs of TQHXD combined with WMT in the treatment of IS. The results showed that TQHXD combined with WMT in the treatment of IS can significantly reduce the patient's NIHSS score, serum Sensitive C-reactive protein, plasma viscosity, serum fibrinogen, serum total cholesterol, and serum triglycerides which can improve the ADL-Barthel score of patients and the effective rate of treatment. But there is no 


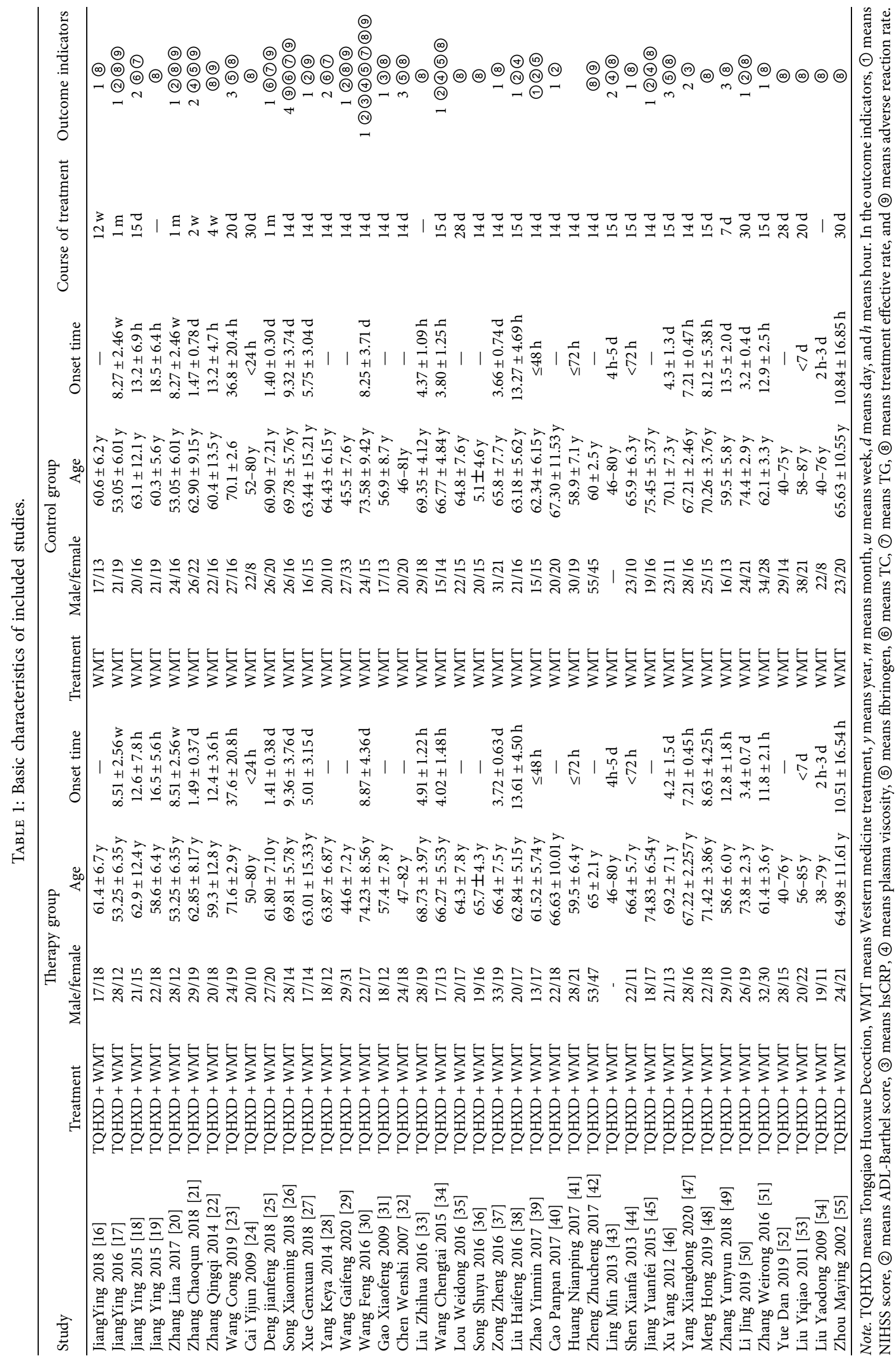




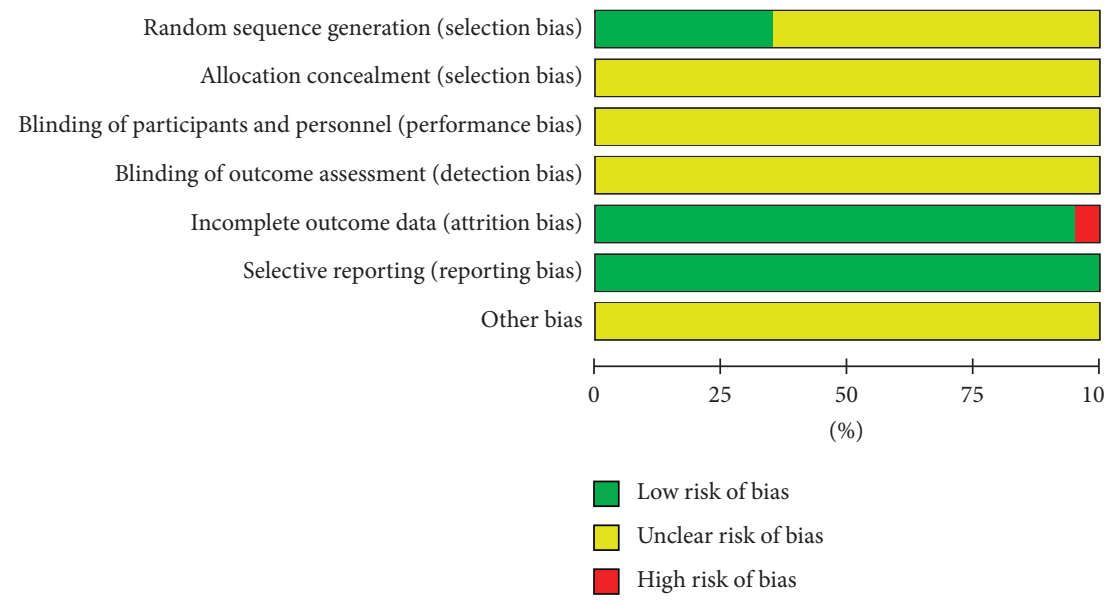

(a)

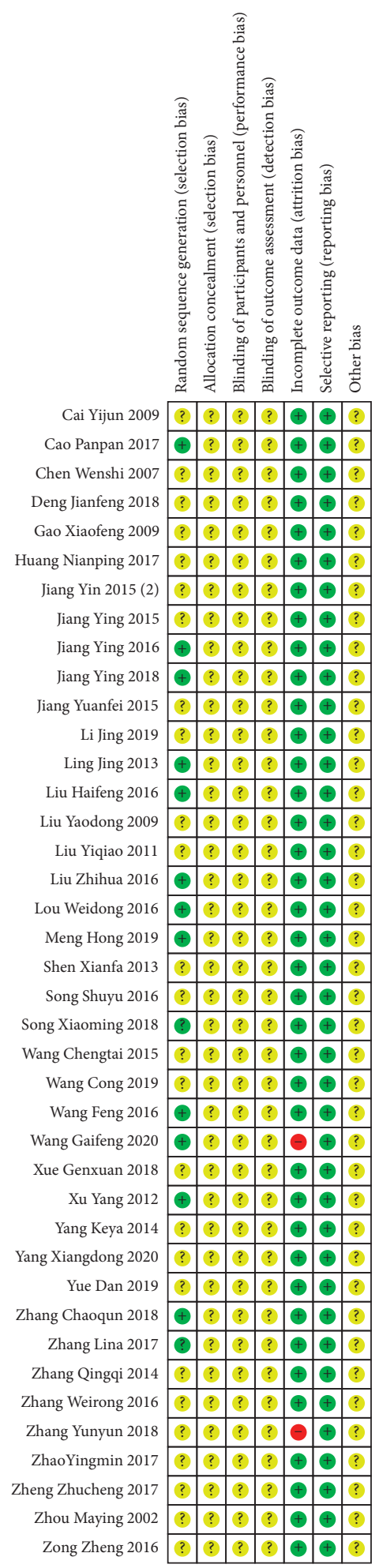

(b)

FIGURE 2: Literature quality evaluation of included studies.

evidence that TQHXD combined with WMT can significantly reduce the incident rate of adverse reactions. $\mathrm{Li}$ Lingfen's study [62] has shown that increased fibrinogen can enhance the aggregation of red blood cells and platelets and that increased levels of fibrinogen can cause increased plasma viscosity, thereby promoting thrombosis and leading to ischemic events. In addition, TC (total cholesterol) and LDL (low-density lipoprotein) are also related to the onset of 


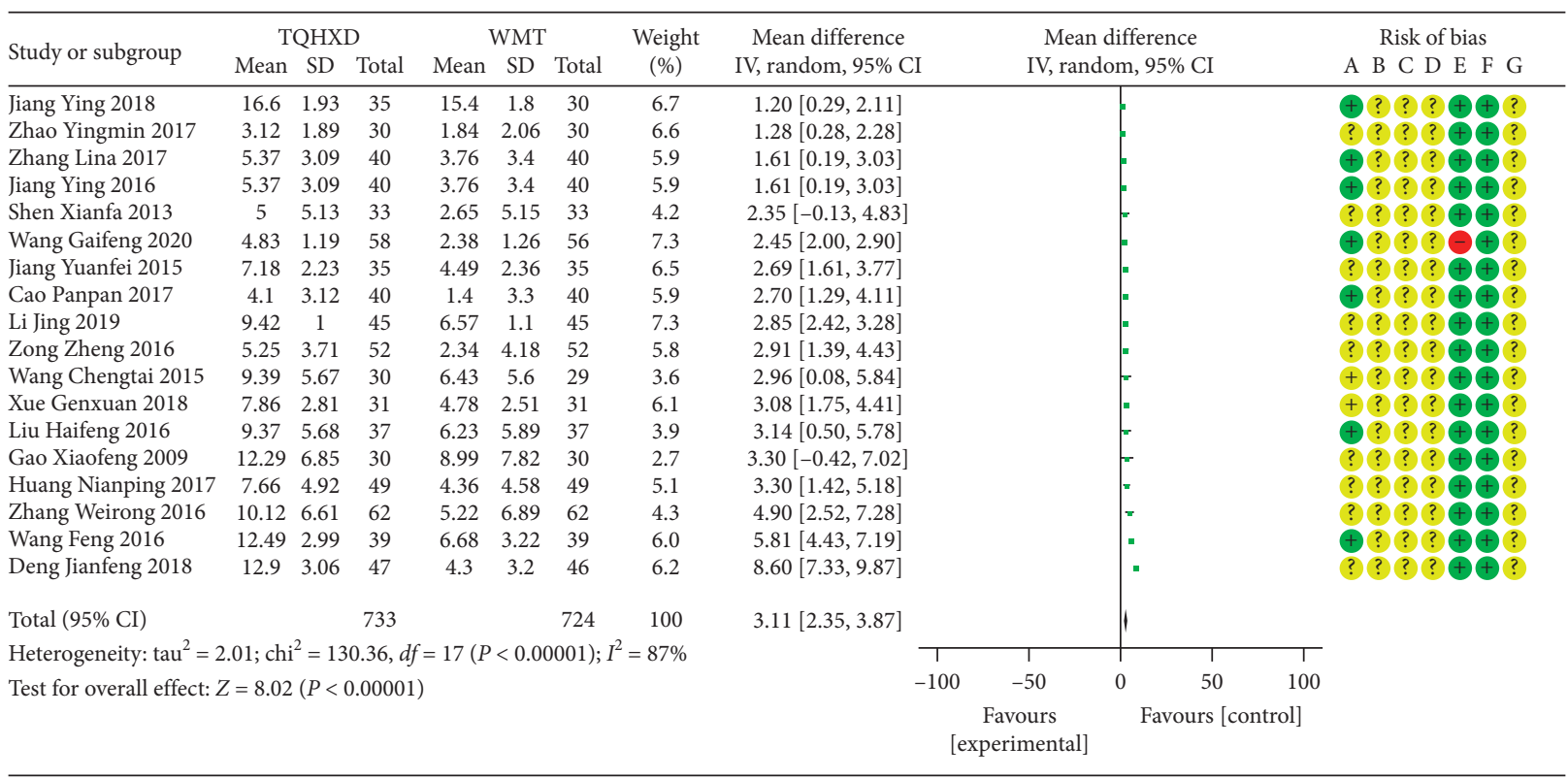

Risk of bias legend
(A) Random sequence generation (selection bias)
(B) Allocation concealment (selection bias)
(C) Blinding of participants and personnel (performance bias)
(D) Blinding of outcome assessment (detection bias)
(E) Incomplete outcome data (attrition bias)
(F) Selective reporting (reporting bias)
(G) Other bias

FIgURE 3: The forest diagram of TQHXD combined with WMT group and WMT group to compare the NIHSS score.

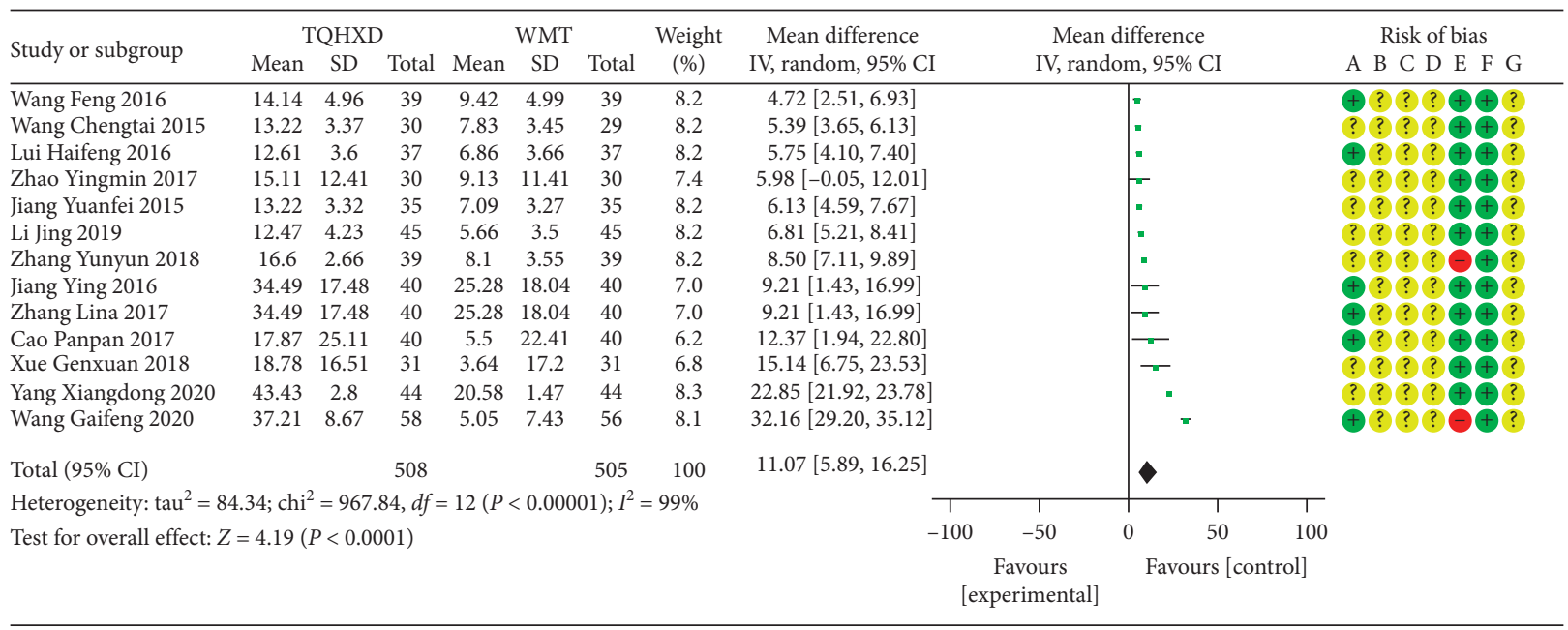

Risk of bias legend
(A) Random sequence generation (selection bias)
(B) Allocation concealment (selection bias)
(C) Blinding of participants and personnel (performance bias)
(D) Blinding of outcome assessment (detection bias)
(E) Incomplete outcome data (attrition bias)
(F) Selective reporting (reporting bias)
(G) Other bias

FIgURE 4: The forest diagram comparing TQHXD combined with WMT group and WMT group to compare the ADL-Barthel score. 


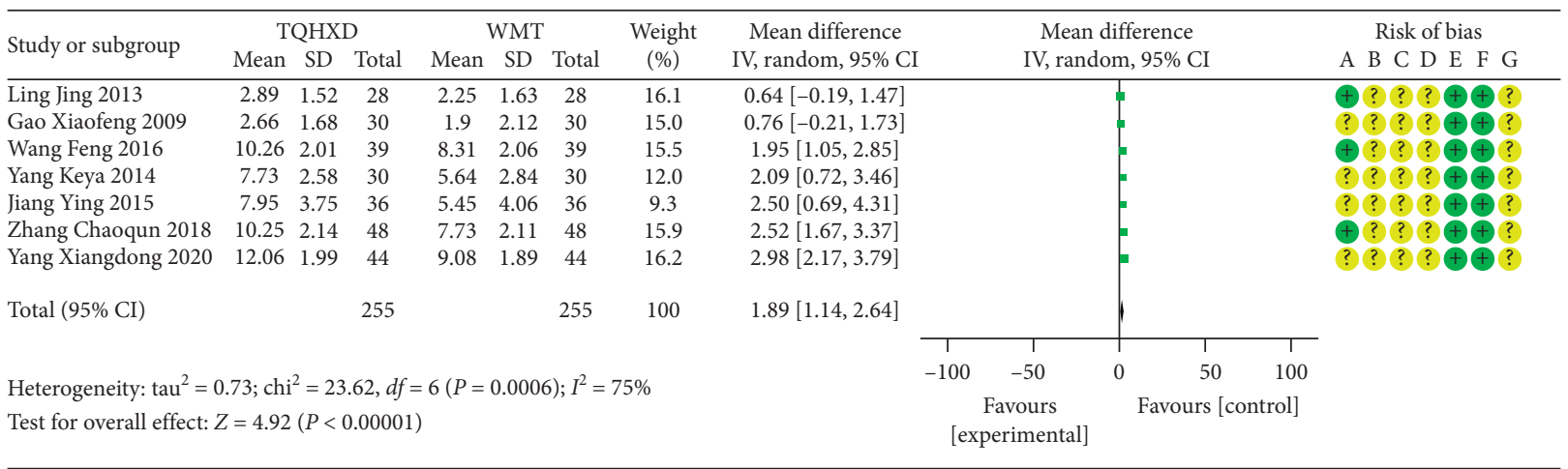

\section{Risk of bias legend}
(A) Random sequence generation (selection bias)
(B) Allocation concealment (selection bias)
(C) Blinding of participants and personnel (performance bias)
(D) Blinding of outcome assessment (detection bias)
(E) Incomplete outcome data (attrition bias)
(F) Selective reporting (reporting bias)
(G) Other bias

FIgURE 5: The forest diagram comparing TQHXD combined with WMT group and WMT group to compare the hsCRP.

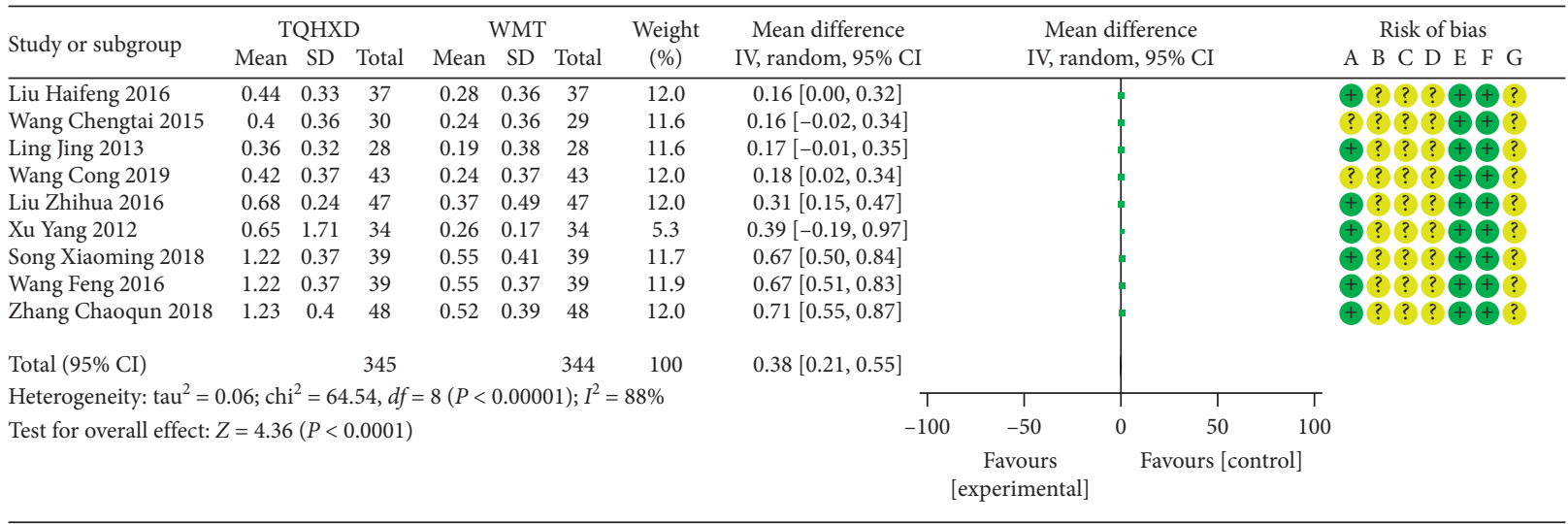

\section{Risk of bias legend}
(A) Random sequence generation (selection bias)
(B) Allocation concealment (selection bias)
(C) Blinding of participants and personnel (performance bias)
(D) Blinding of outcome assessment (detection bias)
(E) Incomplete outcome data (attrition bias)
(F) Selective reporting (reporting bias)
(G) Other bias

FIgURE 6: The forest diagram comparing TQHXD combined with WMT and WMT to compare fibrinogen.

cerebral infarction. Tao Fei's study [63] shows that reducing blood TC, TG, and LDL can prevent and reduce the occurrence of stroke. The existing research results show that TQHXD combined with WMT has an effect on fibrinogen, plasma viscosity, serum total cholesterol, triglycerides, and other indicators, thereby playing a therapeutic effect on IS. Du Yong's study [64] found that gavage of TQHXD can significantly increase the relative expression of LC3II/I, MnSOD, COXIV, and Bcl-2 protein in rats with brain injury and reduce the relative expression of p62 and cleaved caspase-3 protein. The amount indicates that the effect of TQHXD may be related to the activation of mitochondrial autophagy and inhibition of cell apoptosis. The results of adverse reaction analysis showed that there were 28 adverse events in the TQHXD combined with the WMT group and 20 cases in the WMT group. The adverse reactions were mainly dizziness, fatigue, loss of appetite, nausea and vomiting, abdominal pain, and diarrhea. The adverse reaction symptoms were manifested between the two groups, and the difference was not statistically significant $(P>0.05)$. Therefore, it is not believed that the addition of TQHXD will increase the risk of adverse events. In summary, TQHXD combined with WMT has a good effect, which is worthy of application and promotion. 


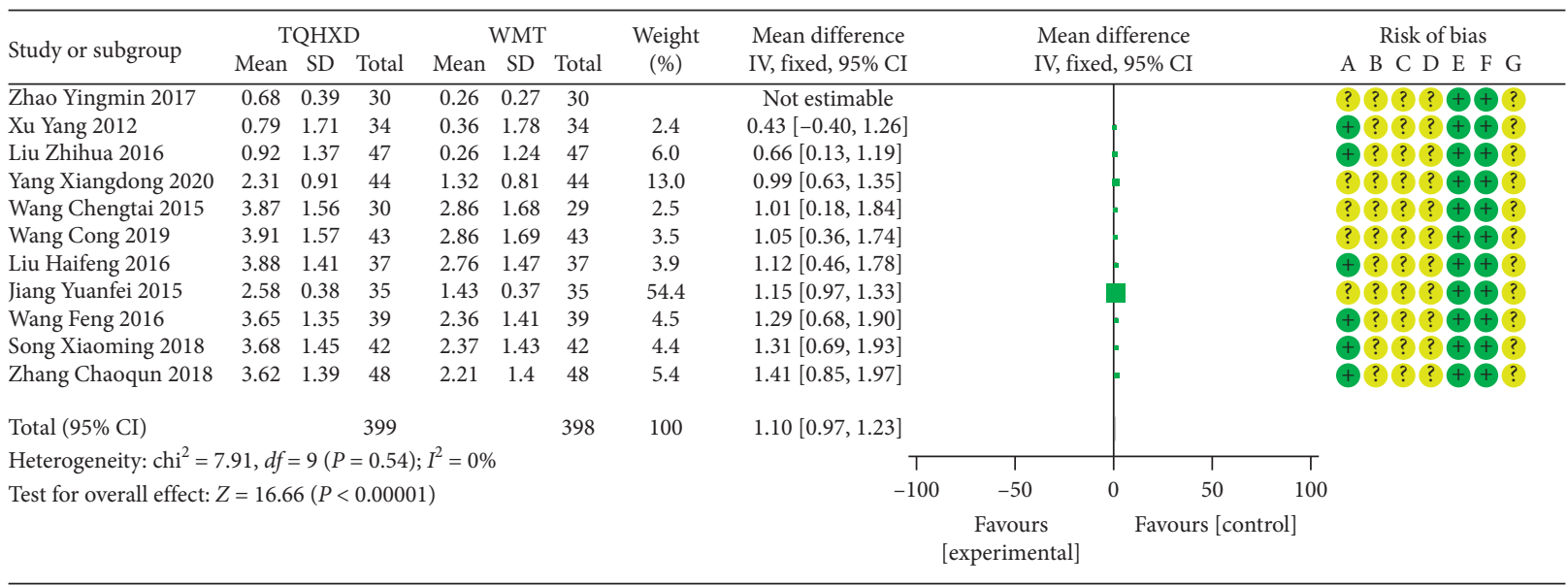

Risk of bias legend

(A) Random sequence generation (selection bias)

(B) Allocation concealment (selection bias)

(C) Blinding of participants and personnel (performance bias)

(D) Blinding of outcome assessment (detection bias)

(E) Incomplete outcome data (attrition bias)

(F) Selective reporting (reporting bias)

(G) Other bias

FIgURE 7: The forest diagram comparing TQHXD combined with WMT and WMT to compare the plasma viscosity.

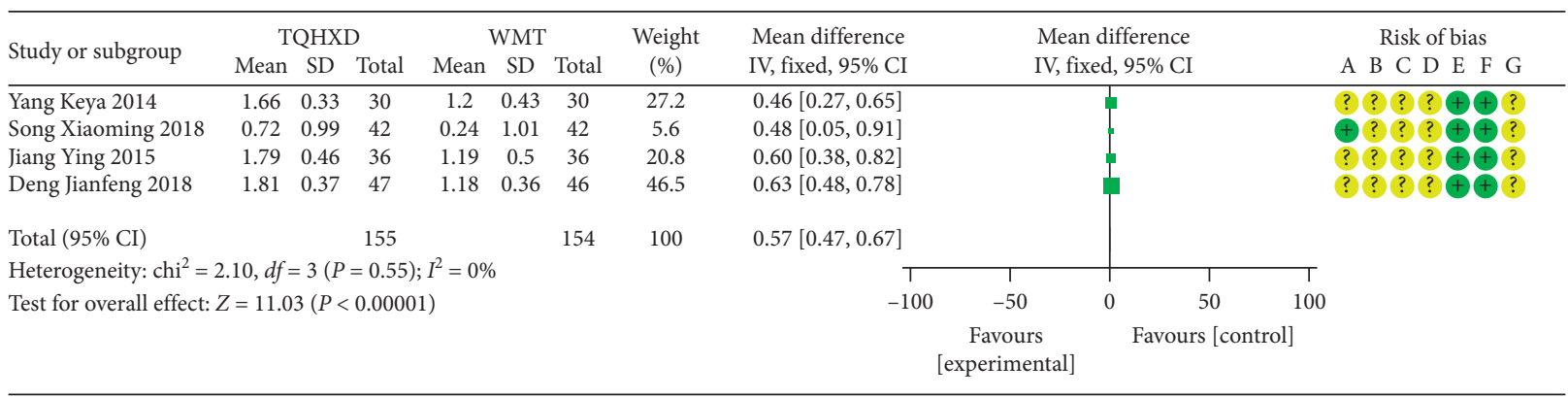

Risk of bias legend
(A) Random sequence generation (selection bias)
(B) Allocation concealment (selection bias)
(C) Blinding of participants and personnel (performance bias)
(D) Blinding of outcome assessment (detection bias)
(E) Incomplete outcome data (attrition bias)
(F) Selective reporting (reporting bias)
(G) Other bias

FIgURE 8: The forest diagram of TQHXD combined with WMT and WMT to compare TC.

\section{Limitation}

However, there were some certain limitations. (1) The quality of the research methodology included in this systematic review is generally poor. The 40 included studies [16-55] did not mention whether blinding was implemented and it is not clear whether there are other biases. (2) The sample size of all studies is small, with only 3260 patients. (3) The adverse reaction reports are not detailed, and the specific symptoms and duration of adverse reactions are not described. (4) The number of studies included in this study is relatively small, where 14 


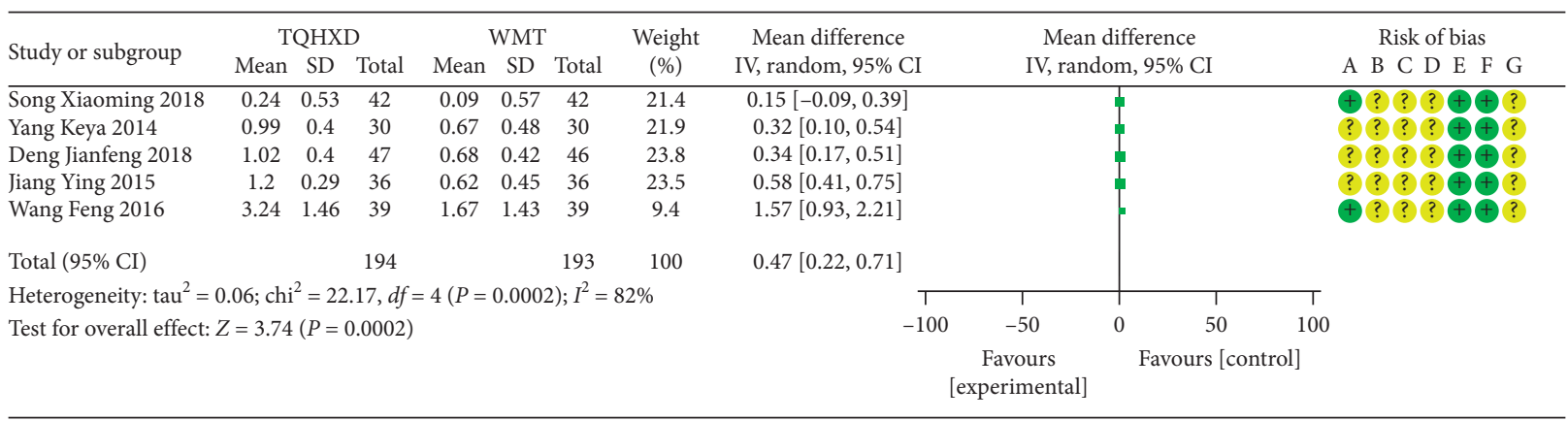

Risk of bias legend

(A) Random sequence generation (selection bias)

(B) Allocation concealment (selection bias)

(C) Blinding of participants and personnel (performance bias)

(D) Blinding of outcome assessment (detection bias)

(E) Incomplete outcome data (attrition bias)

(F) Selective reporting (reporting bias)

(G) Other bias

FIgure 9: The forest diagram of TQHXD combined with WMT and WMT to compare TG.

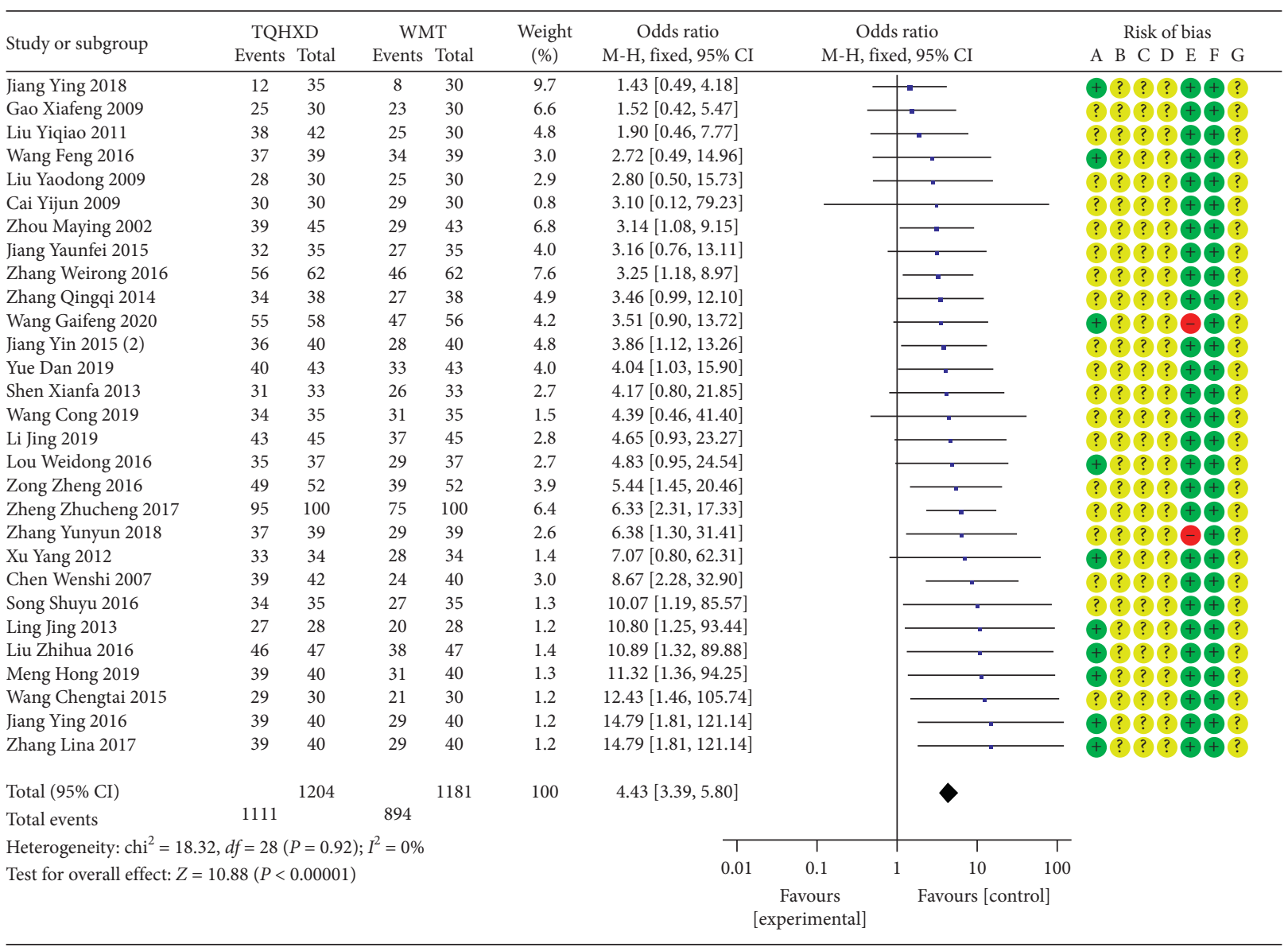

Risk of bias legend
(A) Random sequence generation (selection bias)
(B) Allocation concealment (selection bias)
(C) Blinding of participants and personnel (performance bias)
(D) Blinding of outcome assessment (detection bias)
(E) Incomplete outcome data (attrition bias)
(F) Selective reporting (reporting bias)
(G) Other bias

FIgURE 10: The forest diagram of TQHXD combined with WMT and WMT to compare the treatment efficiency. 


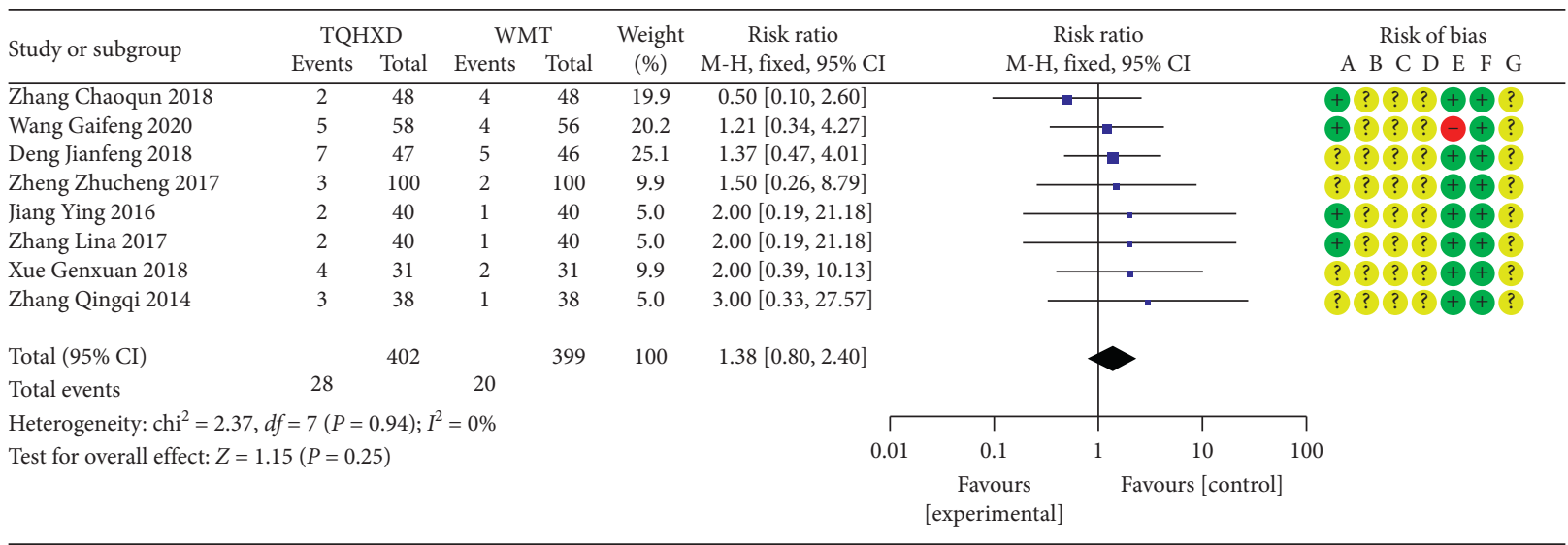

\section{Risk of bias legend}
(A) Random sequence generation (selection bias)
(B) Allocation concealment (selection bias)
(C) Blinding of participants and personnel (performance bias)
(D) Blinding of outcome assessment (detection bias)
(E) Incomplete outcome data (attrition bias)
(F) Selective reporting (reporting bias)
(G) Other bias

FIgURE 11: The forest diagram of TQHXD combined with WMT and WMT to compare the incidence of adverse reactions.

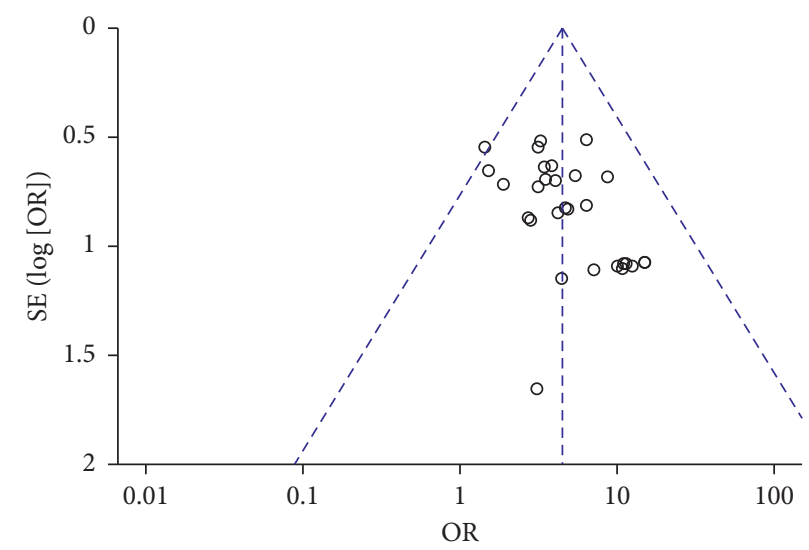

FIgURE 12: The funnel diagram of TQHXD combined with WMT and WMT to compare the treatment efficiency.

studies $[16,17,20,21,26,29,30,33,35,38,40,43,46,48]$ mentioned specific random methods. (5) The number of included studies is small, all included studies are published with positive results, and it is impossible to determine whether there are unpublished negative results.

\section{Conclusion}

In summary, the safety and effectiveness of TQHXD combined with WMT in the treatment of IS still require a large number of RCTs with double-blind trials to verify. However, all studies of TQHXD combined with WMT in the treatment of IS were collected comprehensively, and the clinical efficacy of TQHXD combined with WMT in the treatment of IS was evaluated objectively. Therefore, the results of this study still have certain clinical guiding significance.

\section{Data Availability}

The data used in this article are obtained from public databases. The process including the literature, data extraction, and calculation are all described in the article. If necessary , the first author Da-yuan Zhong (13751728424@163.com) or the corresponding author Yi-hui Deng (644138330@ qq.com) can be contacted to obtain data.

\section{Conflicts of Interest}

The authors declare that they have no conflicts of interest. 


\section{Authors' Contributions}

Da-yuan Zhong and Hao-yue Li contributed equally. Determination of the direction of the topic was done by Yi-hui Deng and Ding-xiang Li; research search was conducted by Ruo-meng Ma and Lan Li; quality evaluation of research was performed by Lan Li and Cheng-ting Jiang; data curation was done by Da-yuan Zhong and Hao-yue Li; writing of the original draft was done by Da-yuan Zhong and Hao-yue Li; article revision was carried put by Yi-hui Deng and Dingxiang Li; article translation was done by Da-yuan Zhong and Yi-hui Deng.

\section{Acknowledgments}

This study was funded by the General Project of National Natural Science Foundation of China (81874416) and Open Fund for First-Class Disciplines of Integrated Chinese and Western Medicine at Hunan University of Traditional Chinese Medicine (2018ZXYJH36).

\section{References}

[1] World Health Organization, Top 10 Global Causes of Deaths, World Health Organization, Geneva, Switzerland, 2016, https://https://www.who,int/en/news-room/fact-sheets/ detail/the-top-10-causes-of-death.

[2] C. O. Johnson, M. Nguyen, G. A. Roth, and E. Nichols, "Global, regional, and national burden of stroke, 1990-2016: a systematic analysis for the global burden of disease study 2016," The Lancet. Neurology, vol. 18, no. 5, pp. 439-458, 2019.

[3] W. Wang, B. Jiang, H. Sun et al., "Prevalence, incidence, and mortality of stroke in China," Circulation, vol. 135, no. 8, pp. 759-771, 2017.

[4] K. Harald, L. Viola, D. Sven et al., "The importance of thrombin in cerebral injury and disease," International Journal of Molecular Sciences, vol. 17, no. 1, p. 84, 2016.

[5] C. -C. Wei, S. -T. Zhang, J. -F. Liu et al., "Association between fibrinogen and leukoaraiosis in patients with ischemic stroke and atrial fibrillation," Journal of Stroke and Cerebrovascular Diseases: The Official Journal of National Stroke Association, vol. 26, no. 11, pp. 2630-2637, 2017.

[6] D. Bushi, M. Ben Shimon, E. Shavit Stein, J. Chapman, N. Maggio, and D. Tanne, "Increased thrombin activity following reperfusion after ischemic stroke alters synaptic transmission in the hippocampus," Journal of Neurochemistry, vol. 135, no. 6, pp. 1140-1148, 2015.

[7] J. Wang, C. Li, T. Chen et al., "Nafamostat mesilate protects against acute cerebral ischemia via blood-brain barrier protection," Neuropharmacology, vol. 105, pp. 398-410, 2016.

[8] M. Yin, Z. Chen, Y. Ouyang et al., "Thrombin-induced, TNFR-dependent miR-181c downregulation promotes MLL1 and NF- $\mathrm{KB}$ target gene expression in human microglia," Journal of Neuroinflammation, vol. 14, no. 1, p. 132, 2017.

[9] S. Wan, Y. Cheng, H. Jin et al., "Microglia activation and polarization after intracerebral hemorrhage in mice: the role of protease-activated receptor-1," Translational Stroke Research, vol. 7, no. 6, pp. 478-487, 2016.

[10] X. An, L. Zhang, Q. Yuan et al., "Overview of the theoretical research on ischemic stroke in traditional Chinese medicine," Guangming Traditional Chinese Medicine, vol. 31, no. 08, pp. 1205-1208, 2016.
[11] N. Cheng and F. Tan, "Discussion on the pathogenesis of blood stasis and early treatment of blood stasis in apoplexy," Chinese Emergency in Traditional Chinese Medicine, vol. 22, no. 10, pp. 1668-1669, 2013.

[12] Y. Jiang, "Effect of Tongqiao Huoxue Decoction on neurotransmitters in rats with ischemic stroke," Chinese Journal of Clinical Pharmacology, vol. 32, no. 14, pp. 1307-1310, 2016.

[13] Y. Jiang, K. Wang, F. Xing et al., "Effects of Tongqiao Huoxue Decoction on neurotrophic factors and oxygen free radicals in rats with cerebral ischemia reperfusion," Genomics and Applied Biology, vol. 37, no. 7, pp. 3085-3093, 2018.

[14] C. Gao, C. Wu, J. Zhao et al., "Guidelines for the diagnosis and treatment of cerebral infarction in China with integrated traditional Chinese and western medicine (2017)," Chinese Journal of Integrated Traditional Chinese and Western Medicine, vol. 38, no. 02, pp. 136-144, 2018.

[15] M. Borenstein, L. V. Hedges, J. P. T. Higgins, and H. R. Rothstein, Introduction to Meta Analysis, vol. 01, p. 17, Science Press, Beijing, China, 2013.

[16] Y. Jiang, K. Wang, Y. Zhang et al., "The effect of Tongqiao Huoxue Recipe combined with flunarizine on the prognosis of patients with Qi deficiency and blood stasis type ischemic stroke," Chinese Journal of Experimental Formulas, vol. 24, no. 11, pp. 156-162, 2018.

[17] Y. Jiang, H. Lu, F. Xing et al., "Curative effect observation of Tongqiao Huoxue Decoction combined with aspirin in the treatment of ischemic stroke," Chinese Journal of Experimental Formulas, vol. 22, no. 08, pp. 187-190, 2016.

[18] Y. Jiang, H. Lu, Y. Zhang et al., "Study on the effect of Tongqiao Huoxue decoction on blood HCY, hs-CRP and blood lipids in patients with acute ischemic stroke," China Journal of Traditional Chinese Medicine, vol. 745, 2016.

[19] Y. Jiang, H. Lu, Y. Zhang et al., "Efficacy of Tongqiao Huoxue decoction in the treatment of ischemic stroke and its effect on the levels of von Willebrand factor and plasma thrombomodulin," China Journal of Chinese Medicine, vol. 2266, 2016.

[20] L. Zhang and Bo Sun, "The effect of Tongqiaohuoxue Decoction combined with aspirin on oxidative stress response and neurological deficit in patients with ischemic stroke," Herald of Chinese Medicine, vol. 23, no. 16, pp. 108-110, 2017.

[21] C. Zhang, "The effect of integrated traditional Chinese and western medicine treatment on hs-CRP level and hemorheology in patients with acute stroke," Henan Medical Research, vol. 27, no. 14, pp. 2583-2584, 2018.

[22] Q. Zhang, "Efficacy of Tongqiao Huoxue Decoction in the treatment of ischemic stroke and its effect on the levels of von Willebrand factor and plasma thrombomodulin," Journal of Pharmaceutical Epidemiology, vol. 23, no. 07, pp. 405-407, 2014.

[23] C. Wang, "Therapeutic effect of Tongqiao Huoxue decoction on cerebral infarction," Heilongjiang Science, vol. 10, no. 08, pp. 38-39, 2019.

[24] Y. Cai, "Tongqiao Huoxue Decoction in the treatment of ischemic stroke," Journal of Chengdu University of Traditional Chinese Medicine, vol. 32, no. 03, pp. 45-46, 2009.

[25] J. Deng, "Clinical observation on 47 cases of acute ischemic stroke treated with integrated traditional Chinese and western medicine," Chinese National Folk Medicine, vol. 27, no. 12, pp. 121-123, 2018.

[26] X. Song, "A randomized parallel controlled study of Jiawei Tongqiao Huoxue Decoction combined with western medicine in the treatment of ischemic stroke (qi deficiency and blood stasis)," Journal of Practical Traditional Chinese Internal Medicine, vol. 33, no. 01, pp. 50-53, 2019. 
[27] G. Xue, "Study on the clinical value of Jiawei Tongqiao Huoxue Decoction in the treatment of acute ischemic stroke," Bethune Medical Journal, vol. 16, no. 06, pp. 618-620, 2018.

[28] K. Yang and Y. Liu, "The effect of Tongqiao Huoxue Decoction on serum HCY, hs-CRP and blood lipids in patients with acute ischemic stroke," Liaoning Journal of Traditional Chinese Medicine, vol. 41, no. 02, pp. 279-280, 2014.

[29] G. Wang, Z. Liu, Ji Lingshan et al., "Clinical observation of Tongqiaohuoxue Decoction combined with stent embolization in the treatment of acute cerebrovascular occlusion," Chinese Journal of Experimental Formulas, vol. 26, no. 06, pp. 79-84, 2020.

[30] F. Wang, W. Wang, X. Wang et al., "Clinical observation of Jiawei Tongqiao Huoxue Decoction in the treatment of acute ischemic stroke," China Emergency in Traditional Chinese Medicine, vol. 25, no. 3, pp. 494-496, 2016.

[31] X. Gao, Y. Zou, D. Zhou et al., "Clinical observation on 60 cases of acute cerebral infarction treated by jiajian Tongqiao Huoxue decoction," Practical Clinical Medicine of Integrated Traditional Chinese and Western Medicine, vol. 9, no. 04, pp. 13-15, 2009.

[32] W. Chen and R. Li, "Tongqiao Huoxue Decoction combined with pancreatic kininogenase to treat acute cerebral infarction," Journal of Bengbu Medical College, vol. 33, no. 04, pp. 439-440, 2008.

[33] Z. Liu, "Analysis of the effect of Tongqiao Huoxue Decoction on patients with cerebral infarction and the influence of hemorheological indexes," Clinical Journal of Chinese Medicine, vol. 8, no. 06, pp. 50-51, 2016.

[34] C. Wang, "Efficacy of Tongqiao Huoxue Decoction in the treatment of acute cerebral infarction and its influence on hemorheology," Journal of Clinical Medicine in Practice, vol. 19, no. 23 , pp. 15-17+27, 2015.

[35] W. Lou, Z. Li, and Z. Xie, "Observation of curative effect of integrated traditional Chinese and western medicine on acute cerebral infarction and its effect on vascular endothelial function," New Chinese Medicine, vol. 48, no. 03, pp. 18-20, 2016.

[36] S. Song, "Efficacy of Tongqiao Huoxue Decoction in the treatment of patients with acute cerebral infarction," Electronic Journal of Integrated Traditional Chinese and Western Medicine Cardiovascular Diseases, vol. 4, no. 16, p. 172, 2016.

[37] Z. Zheng, "Tongqiao Huoxue decoction treated 52 cases of cerebral infarction," Guangming Traditional Chinese Medicine, vol. 31, no. 14, pp. 2042-2044, 2016.

[38] H. Liu, "Effects of Tongqiao Huoxue Decoction combined with conventional western medicine on hemorheology, living ability and nerve function in patients with acute cerebral infarction," Inner Mongolia Traditional Chinese Medicine, vol. 35, no. 13, pp. 75-76, 2016.

[39] Y. Zhao, "Effects of Tongqiao Huoxue Decoction on blood rheology, serum soluble apoptosis-related factors and their ligand levels in patients with acute cerebral infarction," Modern Journal of Integrated Traditional Chinese and Western Medicine, vol. 26, no. 04, pp. 426-428, 2017.

[40] P. Cao and M. Wu, "Clinical study on 40 cases of acute cerebral infarction treated with syndrome of phlegm and blood stasis obstruction by integrated traditional Chinese and western medicine," Jiangsu Traditional Chinese Medicine, vol. 49, no. 06, pp. 26-29, 2017.

[41] N. Huang, Z. Tan, and C. Chen, "Effect of jiawei Tongqiao Huoxue decoction on cerebral collateral circulation in patients with acute cerebral infarction," Journal of Integrated Traditional Chinese and Western Medicine Cardiovascular and Cerebrovascular Diseases, vol. 15, no. 17, pp. 2169-2171, 2017.

[42] Z. Zheng, "Efficacy analysis of Tongqiao Huoxue Decoction combined with edaravone injection in the treatment of acute cerebral infarction," Chinese Folk Therapy, vol. 25, no. 11, pp. 59-60, 2017.

[43] L. Jing, "Efficacy observation on the treatment of acute cerebral infarction with Tongqiao Huoxue decoction," World Chinese Medicine, vol. 8, no. 03, pp. 303-305, 2013.

[44] X. Shen, "Clinical observation on the treatment of acute cerebral infarction with Tongqiao Huoxue decoction," Sichuan Traditional Chinese Medicine, vol. 31, no. 12, pp. 95-96, 2013.

[45] Y. Jiang, "Clinical observation of Tongqiao Huoxue Decoction in the treatment of acute cerebral infarction," China Emergency in Traditional Chinese Medicine, vol. 24, no. 03, pp. 549-550, 2015.

[46] Xu Yang and D. Wang, "Efficacy observation of Tongqiao Huoxue Decoction in the treatment of acute cerebral infarction," Chinese and Foreign Medical Research, vol. 10, no. 08, pp. 71-72, 2012.

[47] X. Yang, "Analysis of clinical efficacy of Tongqiao Huoxue Decoction in the treatment of acute cerebral infarction," Psychology Monthly, vol. 15, no. 01, p. 221, 2020.

[48] H. Meng, "Clinical effect of Tongqiao Huoxue Decoction on acute cerebral infarction," China Practical Medicine, vol. 14, no. 35, pp. 158-159, 2019.

[49] Y. Zhang, "Tongqiao Huoxue Decoction in the auxiliary treatment of acute cerebral infarction," Inner Mongolia Traditional Chinese Medicine, vol. 37, no. 07, pp. 24-25, 2018.

[50] Li Jing, "Observation on the clinical efficacy of Tongqiaohuoxue Decoction combined with western medicine in the treatment of acute cerebral infarction," Clinical Journal of Chinese Medicine, vol. 11, no. 15, pp. 36-37, 2019.

[51] W. Zhang and Y. Zhang, "Tongqiao Huoxue Decoction in the treatment of acute cerebral infarction," Massage and Rehabilitation Medicine, vol. 7, no. 02, pp. 70-71, 2016.

[52] D. Yue, "Clinical observation of Tongqiao Huoxue decoction in treating acute cerebral infarction with diabetes," Modern Distance Education of Chinese Medicine, vol. 17, no. 14, pp. 50-52, 2019.

[53] Y. Liu and Z. Xu, "Tongqiao Huoxue decoction treated 80 cases of cerebral infarction," Guangming Traditional Chinese Medicine, vol. 26, no. 02, pp. 255-257, 2011.

[54] Y. Liu, L. Sun, J. Ma, and H. Duan, "Efficacy of lubigre and Tongqiao Huoxue decoction in the treatment of acute cerebral infarction," Chinese Ethnic Folk Medicine, vol. 19, no. 01, p. 126, 2010.

[55] M. Zhou and B. Xu, "Clinical observation of jiajian Tongqiao Huoxue decoction in the treatment of cerebral infarction," Chinese Journal of Medical Writing, vol. 9, no. 23, pp. 2023-2024, 2002.

[56] W. H. Hsu, Y. C. Shen, Y. J. Shiao et al., "Combined proteomic and metabolomic analyses of cerebrospinal fluid from mice with ischemic stroke reveals the effects of a Buyang Huanwu decoction in neurodegenerative disease," PLoS One, vol. 14, no. 1, Article ID e0209184, 2019.

[57] P. Gulati, N. Singh, and A. Muthuraman, "Pharmacologic evidence for role of endothelial nitric oxide synthase in neuroprotective mechanism of ischemic postconditioning in mice," Journal of Surgical Research, vol. 188, no. 1, pp. 349-360, 2014.

[58] J. Peng, "Study on the efficacy and safety of the clinical treatment model of acute ischemic cerebrovascular disease in 
cerebral angiography," Digest of World Latest Medical Information, vol. 17, no. 63, p. 12+14, 2017.

[59] L. Wang, "Clinical study of arterial thrombolysis combined with interventional methods for the treatment of elderly patients with acute cerebrovascular occlusion," Modern Diagnosis and Treatment, vol. 28, no. 10, pp. 1872-1873, 2017.

[60] J. Hu, W.-S. Pang, J. Han, K. Zhang, J.-Z. Zhang, and L.-D. Chen, "Gualou Guizhi decoction reverses brain damage with cerebral ischemic stroke, multi-component directed multi-target to screen calcium-overload inhibitors using combination of molecular docking and protein-protein docking," Journal of Enzyme Inhibition and Medicinal Chemistry, vol. 33, no. 1, pp. 115-125, 2018.

[61] K. Chen, L. Li, and W. Weng, "Study on blood stasis syndrome and promoting blood circulation to remove blood stasis," Journal of Integrated Traditional Chinese and Western Medicine Cardio-Cerebrovascular Disease, vol. 3, no. 1, pp. 1-2, 2005.

[62] L. Li, "Hemorheology detection and clinical significance," Modern Journal of Integrated Traditional Chinese and Western Medicine, vol. 14, no. 18, pp. 2480-2482, 2005.

[63] T. Fei and S. Ju, "Evaluation of cerebral infarction and risk factors," Digest of World Latest Medical Information, vol. 19, no. 05, pp. 29-30, 2019.

[64] Y. Du, G. Wang, S. Zhang et al., "Gavage of Tongqiao Huoxue Decoction on the improvement of brain injury in mice and its mechanism," Shandong Medicine, vol. 60, no. 9, pp. 48-52, 2020 . 